\title{
Alterations in PGC1a expression levels are involved in colorectal cancer risk: a qualitative systematic review
}

\author{
Jéssica Alonso-Molero ${ }^{1,2,5^{*}}$ (D), Carmen González-Donquiles ${ }^{1}$, Tania Fernández-Villa' ${ }^{1}$, Fernanda de Souza-Teixeira ${ }^{1,3}$,
} Laura Vilorio-Marqués ${ }^{1}$, Antonio J. Molina ${ }^{1}$ and Vicente Martín ${ }^{1,4}$

\begin{abstract}
Background: Colorectal cancer (CRC) is a major global public health problem and the second leading cause of cancer-related death. Mitochondrial dysfunction has long been suspected to be involved in this type of tumorigenesis, as supported by an accumulating body of research evidence. However, little is known about how mitochondrial alterations contribute to tumorigenesis. Mitochondrial biogenesis is a fundamental cellular process required to maintain functional mitochondria and as an adaptive mechanism in response to changing energy requirements. Mitochondrial biogenesis is regulated by peroxisome proliferator-activated receptor gamma coactivator 1-a (PPARGC1A or PGC1a). In this paper, we report a systematic review to summarize current evidence on the role of PGC1a in the initiation and progression of CRC. The aim is to provide a basis for more comprehensive research.
\end{abstract}

Methods: The literature search, data extraction and quality assessment were performed according to the document Guidance on the Conduct of Narrative Synthesis in Systematic Reviews and the PRISMA declaration.

Results: The studies included in this review aimed to evaluate whether increased or decreased PGC1a expression affects the development of CRC. Each article proposes a possible molecular mechanism of action and we create two concept maps.

Conclusion: Our systematic review indicates that altered expression of PGC1 a modifies CRC risk. Most studies showed that overexpression of this gene increases CRC risk, while some studies indicated that lower than normal expression levels could increase CRC risk. Thus, various authors propose PGC1 a as a good candidate molecular target for cancer therapy. Reducing expression of this gene could help to reduce risk or progression of CRC.

Keywords: PGC1a or PPARGC1a, Colorectal cancer (CRC), Signaling or metabolic pathways, Molecular mechanism

\section{Background}

Colorectal cancer (CRC) is a major global public health problem and the second leading cause of cancer-related death. It is the third most commonly diagnosed cancer in men and the second in women [1]. CRC is the seventh and fourth most common cause of death and loss of life expectancy in Western Europe, respectively, and is associated with an elevated consumption of resources $[2,3]$.

\footnotetext{
* Correspondence: jalonm00@estudiantes.unileon.es

${ }^{1}$ Grupo de Investigación en Interacciones Gen-Ambiente y Salud, Universidad de León, León, Spain

${ }^{2}$ Universidad de Cantabria, Santander, Spain

Full list of author information is available at the end of the article
}

Mitochondrial dysfunction has long been suspected to be involved in this type of tumorigenesis, as supported by an accumulating body of research evidence. However, little is known about how mitochondrial alterations contribute to tumorigenesis [4-7]. Mitochondrial biogenesis is a fundamental cellular process required to maintain functional mitochondria and as an adaptive mechanism in response to changing energy requirements [8]. Both endogenous and exogenous factors, as well as numerous signaling pathways and gene expression patterns, converge upon the mitochondrial biogenesis process to coordinate the energy needs of cells, tissues and the entire organism $[4,8,9]$. 
The master regulator of mitochondrial biogenesis is peroxisome proliferator-activated receptor gamma coactivator 1- $\alpha$ (PPARGC1A or PGC1 $\alpha$ ), because it controls production of mitochondrial proteins [10]. This gene is a transcriptional coactivator of the PGC-1 (peroxisome proliferator-activated receptor gamma coactivator 1) gene family, which has three known members, PGC1 $\alpha$, PGC1 $\beta$ and PRC (PGC-1 related coactivator). PGC1 $\alpha$ and PGC1 $\beta$ are expressed in tissues with high energy demand, while PRC is expressed ubiquitously $[10,11]$. While all three members of this family are potent regulators of mitochondrial function and biogenesis, PGC1 $\alpha$ is the most widely studied, and the other two are less well characterized [8, 11-13]. However, [11, 14].

PGC1 $\alpha$ acts as a master regulator of energy metabolism and mitochondrial biogenesis by integrating and coordinating the activity of other transcription factors, such as Nuclear respiratory factor 1, Nuclear factor 2, PPAR $\alpha$ (peroxisome proliferator-activated receptors $\alpha$ ) and Mitochondrial transcription factor A [15]. Various endogenous and exogenous factors also regulate mitochondrial biogenesis through this gene $[4,9]$. In addition, expression levels of PGC1 $\alpha$ appear to be directly related to mitochondrial biogenesis activity. As a multi-response factor, many agents and events regulate PGC1 $\alpha$ expression via multiple intracellular mediators [16].

Several mutations in nuclear and mitochondrial genes encoding for mitochondrial components have been reported to be associated with increased cancer risk [17], and mitochondrial loss is known to precede the development of dysplasia [6]. We believe that there is a clear relationship between $\mathrm{CRC}$ and $\mathrm{PGC} 1 \alpha$; however, the role of mitochondria and PGC1 $\alpha$ in CRC is poorly understood at present.

Several studies suggest that $\mathrm{PGC} 1 \alpha$ and related genes can regulate different pathways, such as mitochondrial biogenesis, antioxidant systems, reactive oxygen species, de novo lipid synthesis, and glycolysis, there by playing a role in risk for and development of CRC (See discussion) [10, 11]. Although there is no widely accepted mechanism to explain how PGC1 $\alpha$ is involved in human CRC, it is essential to understand this mechanism in order to reduce CRC risk, as well as the development of novel therapeutic tools to treat tumors and to support measures to reduce CRC risk.

In this paper, we report a systematic review to summarize current evidence on the role of PGC1 $\alpha$ in the initiation and progression of CRC. Since there is a limited evidence on this specific question, we used a broad, inclusive search strategy, with the aim of providing a basis for more comprehensive investigation.

\section{Methods}

\section{Search strategy}

The literature search, data extraction and quality assessment were performed according to the document Guidance on the Conduct of Narrative Synthesis in Systematic Reviews [18] and the PRISMA declaration [19]. This search was made between June and September of 2016.

We conducted a web-based search of 8 databases (Cochrane library, PubMed, Scopus, Web of Science, PsycINFO, Scielo, PLoS One and PubMedCentral (PMC)), using the following search terms: "Colorectal OR Colon OR Rectum OR Rectal" and "Cancer OR Carcinoma OR Tumor OR Tumour OR Neoplasm OR Cancer Cells" and "Mitochondrial Biogenesis OR Mitochondrial dysfunction OR Mitochondria OR Mitochondrion" and "Warburg effect" and "OXPHOS OR Oxidative phosphorylation OR Anaerobic glycolysis" and "PGC1A OR PPARGC1A OR Peroxisome Proliferatoractivated Receptor gamma coactivator 1 alpha". The search was restricted to English language articles. We used this quite broad search strategy because of the paucity of research on this specific issue.

\section{Selection process}

The list of articles obtained by this search was manually screened to identify relevant articles. We first read the titles and removed irrelevant articles, and then read the abstracts to eliminate those not directly related to the objective of this review. We imported the resulting set of articles into a reference management program (Endnote), which allowed us to detect duplicate articles. Finally, we read the full text and decided if the article should be included in this review according to the exclusion criteria described below.

\section{Study selection}

We applied the following exclusion criteria during each stage of the selection process mentioned above:

1) Title - We eliminated articles that did not deal with cancer or inflammatory bowel diseases (e.g. Ulcerative Colitis or Crohn's disease, see below). We also excluded articles dealing with genes other than PGC1 $\alpha$ or PGC1 $\beta$ (there is evidence that both have a similar role in the organism).

2) Abstract - We eliminated articles that did not directly deal with CRC or inflammatory bowel diseases that ultimately develop into CRC, or did not deal with some isoform of PGC1 ( $\alpha$ or $\beta$ ), or with related pathways.

3) Full-text - i) We included articles on basic research such as with cell lines or animals. For human studies, we included both basic research, and population-based observational studies. ii) We included articles on colorectal cancer, as well as those on other diseases, such as 
ulcerative colitis, where these ultimately deal with CRC. We did not consider demographic factors during study selection because we considered a broad range of study types, including basic research.

4) The online search was replicated independently by two reviewers, who reviewed and filtered the titles, and created a draft list of titles. This list of articles to be included was agreed upon by three people (with the first author) based on the exclusion/inclusion criteria. The reviewers then independently reviewed the full text of the articles according to the inclusion criteria. Discrepancies were discussed and resolved in collaboration with the principal investigator.

\section{Preliminary synthesis}

We used tabulation and visual representations of data to reduce studies to their key characteristics, which could be important for understanding the objective of this review.

\section{Relationship between papers Evaluating heterogeneity}

In general terms, this technique focused on the characteristics of the various studies and their potential relationship with the findings. The following characteristics were assessed:

- Cells lines (human or animals $=1$ )

- Colorectal cancer (Inflammatory bowel diseases as previous disease $=1$ )

- PGC1 $\alpha$ or PGC1 $\beta$ expression

- Relationship with other genes

- reactive oxygen species

- Mitochondrial biogenesis

- Chemotherapy

These characteristics were scored as 1 if they were present and 0 if absent. We took the sum of these values to obtain a picture of the heterogeneity of the articles in this review. Using these values, we created a comparison graph to evaluate the shared features.

\section{Idea webbing and concept mapping [18]}

Idea webbing is a method for conceptualizing and exploring connections among the findings reported by the studies included in the review (data not shown). Using idea webbing we obtained a concept map, a visual picture linking multiple pieces of evidence across several studies. The aim was to construct a model of key concepts related to PGC1 $\alpha$ and PGC1 $\beta$, and to represent the relationships between these and the development of CRC.

\section{Checking the synthesis with authors of primary studies}

We compared our results to those of other systematic reviews to support our ideas.

\section{Results}

\section{Literature search results}

Using the search terms described in the Methods section, but excluding the Boolean operators, we identified 7688 manuscripts from our search of PubMed, Scopus, Web Of Science (WOS), PsycInfo, Cochrane, Scielo and PLoSOne (no results were returned by PsycInfo or Cochrane). When the Boolean operators were included, 214 articles were returned (Fig. 1). We retained and analyzed 34 papers that met the eligibility criteria described in the Methods section. Of these 34 abstracts, 15 fulltext articles were retrieved for detailed evaluation and 12 studies were included in the final analysis. Figure 1 illustrates the article screening and selection process.

Eighteen articles were excluded for the following reasons: i) Article mentions other genes related to PGC1 $\alpha$, but does not deal with PGC1 $\alpha$ itself. ii) Article mentions PGC1 $\alpha$, but does not deal with CRC. iii) Article mentions PGC1 $\alpha$ and its relationship with cancer, but does not deal with CRC. iv) Article mentions PGC1 $\alpha$ and its relationship with inflammatory bowel diseases, but does not deal with CRC. v) Article mentions PGC1 $\alpha$ and colorectal cancer but not define a relationship between them. A full summary of these data is shown in Additional file 1: Table S1 online.

\section{Preliminary synthesis}

We chose tabulation to visually represent the data, with the aim of reducing studies to the key characteristics that could be important for understanding the relationship between PGC1 $\alpha$ and CRC. The key message observed in these articles was that patterns of altered PGC1 $\alpha$ expression affect risk and development of CRC via various molecular mechanisms, including mitochondrial biogenesis, antioxidant systems, reactive oxygen species, de novo lipid synthesis, glycolysis and alterations in the expression of other genes. Most of this information was obtained from basic research, such as cell culture, since there is little information about this field. These data are summarized in Table 1, and full details are provided in Additional file 1: Table S2 online. In addition, Table 2 summarizes the original material used in each of these articles.

\section{Relationships between papers Heterogeneity}

We analyzed differences in relevant characteristics between the selected articles studied using the graph shown in Fig. 2. 


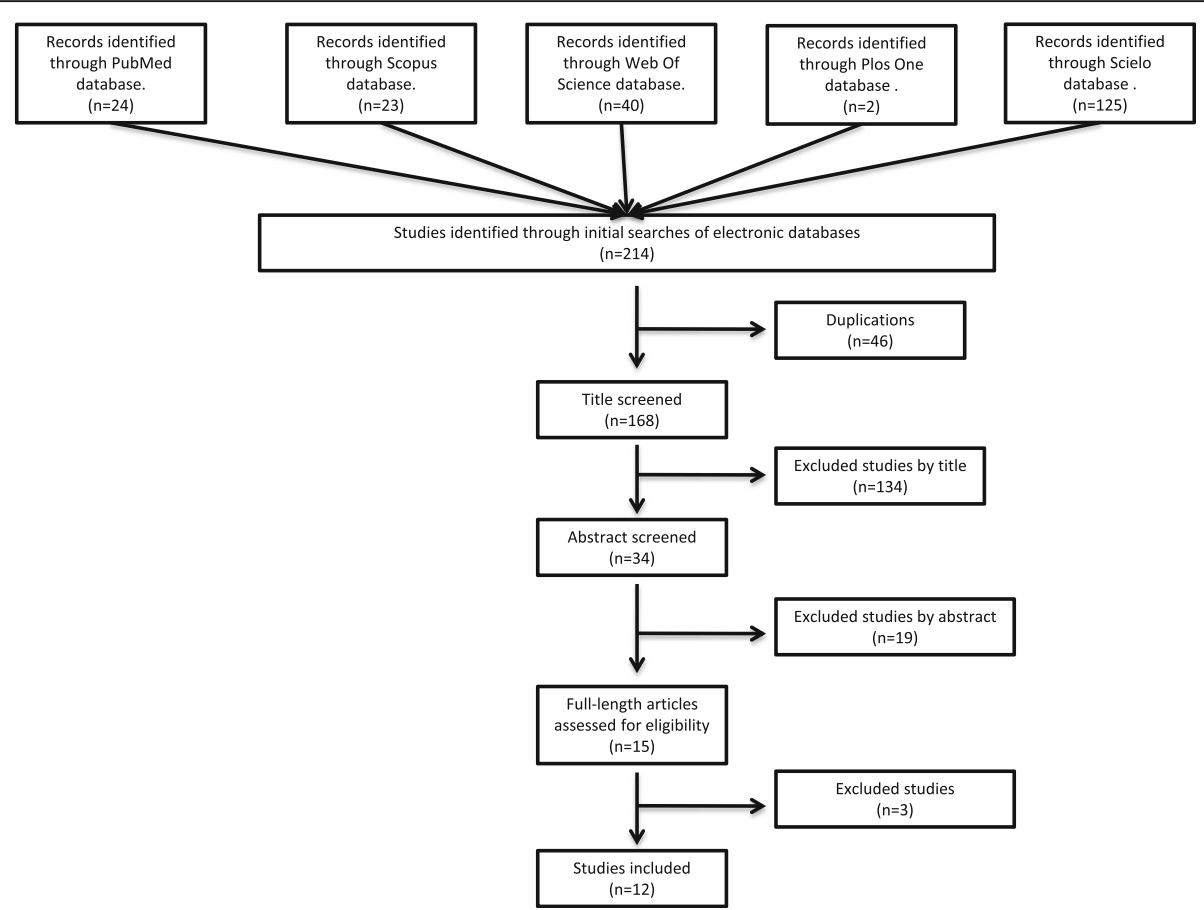

Fig. 1 Summary of article selection process

We assessed the qualitative outcomes of the 12 studies in terms of 6 features: study of CRC; assessment of PGC1 $\alpha$ or PGC1 $\beta$ (which should be present in all papers); relationship between PGC1 $\alpha$ and another gene; test for presence of reactive oxidative species; study of mitochondrial biogenesis; study of chemotherapy. Four papers (30.8\%) had a maximum score of five points, and another 4 articles $(30.8 \%)$ had a score of four points. One study $(7.6 \%)$ had three points, two (15.4\%) had two points, and another two (15.4\%) had six points. These proportions show that the studies were sufficiently homogeneous to perform a systematic narrative review.

\section{Concept mapping}

Based on the information in Table 1, we performed idea webbing for each article, from which we obtained two concept maps. The first concept map is based on D'Errico et al. [20], whose aim was to show that PGC1 $\alpha$ is highly expressed on the surface of the intestinal epithelium but is poorly expressed in the crypts, and is also reduced in intestinal tumors. D'Errico et al. analyzed the expression and function of PGC1 $\alpha$ along the crypt-tovillus axis under normal conditions, and observed that PGC1 $\alpha$ is poorly expressed in the proliferative compartment at the bottom of the crypts, but, conversely, is highly expressed at the villus tips, promoting mitochondria-mediated apoptosis via the accumulation of reactive oxygen species (Fig. 3a).
These authors also identified that overexpression of PGC1 $\alpha$ in human colon cancer cells (HT29) activates metabolic changes such as mitochondrial activation, which produce a proapoptotic effect via reactive species oxygen accumulation (Fig. 3b). Thus, they conclude that PGC1 $\alpha$ is a metabolic regulator of intestinal cell fate and protects against tumorigenesis.

The second concept map highlights patterns of altered gene expression in the development of colorectal cancer. Most papers included in this concept map suggest that PGC1 $\alpha$ expression is increased during the development of $\mathrm{CRC}$, while reduced PGC1 $\alpha$ expression reduces risk and progression of this disease. Nonetheless, some studies have reported the opposite, that reduced PGC1 $\alpha$ expression levels can also increase cancer risk (Fig. 4).

Figure 4 shows various possible mechanisms of action of PGC1 $\alpha$ in relation to CRC development, and suggests four different mechanisms via which this gene can become overexpressed. First, high expression of PGC1 $\alpha$ seems to increase Antioxidant systems, which reduces the number of reactive oxygen species, resulting in inhibition of apoptosis. Second, overexpression of PGC1 $\alpha$ induces the mitochondrial biogenesis pathway, increasing cellular growth (proliferation). Third, overexpression of PGC1 $\alpha$ induces glucose uptake, increasing cell proliferation. Fourth, high levels of PGC1 $\alpha$ could reduce glycolysis and increase oxidative phosphorylation, which increases cells' resistance to chemotherapy. In the map, we can observe certain factors that trigger overexpression of $\mathrm{PGC} 1 \alpha$, such as the 
Table 1 Summary of the key characteristics of the selected studies

\begin{tabular}{|c|c|c|c|c|}
\hline Title & First Author & Year & Primary results & Conclusion \\
\hline $\begin{array}{l}\text { Overexpression of PGC1-alpha } \\
\text { enhances cell proliferation and } \\
\text { tumorigenesis of HEK293 cells through } \\
\text { the upregulation of Sp1 and Acyl-CoA } \\
\text { binding protein. }\end{array}$ & Sung-Won Shin & 2014 & $\begin{array}{l}\text { 1) PGC-1a accelerates proliferation of } \\
\text { HEK293 and CT-26 cells. 2) Knockdown } \\
\text { of PGC1-a expression results in decreased } \\
\text { cell proliferation of human colorectal } \\
\text { cancer cells. 3) PGC-1a promotes the } \\
\text { oncogenic potential of HEK293 cells. } \\
\text { 4) PGC-1aoverexpressing HEK293 cells } \\
\text { have decreased sensitivity to oxidative } \\
\text { stress. }\end{array}$ & $\begin{array}{l}\text { PGC-1a overexpression upregulates } \\
\text { proliferation of HEK293 and CT26 } \\
\text { cells. In addition, this expression } \\
\text { correlates with enhanced } \\
\text { tumorigesis. Moreover, PGC-1a siRNA } \\
\text { transfection resulted in decreased } \\
\text { cell proliferation. Further studies to } \\
\text { clarify the molecular interactions are } \\
\text { needed. }\end{array}$ \\
\hline
\end{tabular}

PGC-1 $\beta$ promotes enterocyte lifespan Elena Bellafante and tumorigenesis in the intestine

Mitochondria and Tumor Progression in Ulcerative Colitis

PGC1a promotes tumor growth by inducing gene expression programs supporting lipogenesis.

Bax is necessary for PGCla proapoptotic effect in colorectal cancer cells

PGC-1 1 / $\beta$ upregulation is associated with improved oxidative phosphorylation in cells harboring nonsense mtDNA mutations

Cigdem

Himmetoglu

Ussakli

Kavita Bhalla

Ilenia D’Errico

Sarika Srivastava
2011 1) Loss of PGC1 protects against both colon and liver tumorigenesis. 2) Overexpression PGC1a promotes tumor growth in vivo. 3) PGCla mediated induction of fatty acid synthesis promotes tumor growth.

2011 1) PGC1a induces Bax activation. 2) PGC1a increases mitochondrial activity. 3) PGC1a induces apoptosis in the presence of Bax, but not without Bax. 4) PGC1a inhibits tumor growth in presence of Bax.

2007 1) PGC-1a and PGC-1 $\beta$ are markedly upregulated in V425. 2) Overexpression of PGC-1a and PGC-1 $\beta$ transcriptional coactivators stimulates mitochondrial respiration, at least in osteosarcoma cybrids. 3) Overexpression of PGC-1a stimulates complex IV activity. 4 ) Overexpression of PGC-1a/ $\beta$ transcriptional coactivators can stimulate respiration in oxidative phosphorylation-deficient cells.

Validation of the Use of DNA Pools and Primer Extension in Association Studies of Sporadic Colorectal Cancer for Selection of Candidate SNPS
Mette Gaustadnes

Results were analyzed using the $x 2$ test with a level of significance $a=0.05$.

Five SNPs were found. The SNP analysis of the $\left.{ }^{*} 604517\right) 3^{\prime}$ utr96516 was not reproducible, but it was always statistically significant.
PGC-1 $\beta$ seems to act as an adaptive self-point regulator, capable of providing a balance between mitochondrial activity and production of increased reactive oxygen species.

1) At the biomarker level, COX loss precedes tumor progression in UC. 2) At the biological level, the loss of COX represents a reduction in the number of mitochondria in preneoplasia, which is restored in cancers. It appears to be driven by PGC1a.

1) Novel role for PGC1a in promoting carcinogenesis and tumor growth. 2) PGCla coordinates the induction of a gene expression program that facilitates the conversion of glucose to fatty acids. 3) PGC1a is a potential therapeutic target for chemoprevention.

1) In the presence of Bax, the PGC1a-induced accumulation of reactive oxygen species is one of the main apoptosis-driving factors in CRC cells. 2) PGC1a is able to induce Bax activation and translocation to mitochondria, thus leading to apoptotic cascade.

1) In V425 cells, the

Ca2 + -dependent signaling events are active for relatively longer periods, which in turn might activate the nuclear genes (including PGC$1 \mathrm{a} / \beta$ ) involved in tumor invasion and metastasis. 2) Overexpression of PGC-1 $1 / \beta$ can stimulate respiration in oxidative phosphorylation deficient cells. 3) This pathway could be explored as a therapeutic approach for the treatment of human mitochondrial diseases.

The results of this article allow us to conclude that the difference between cases and controls would be statistically significant for $n=600$ cases and $n=600$ controls. 
Table 1 Summary of the key characteristics of the selected studies (Continued)

\begin{tabular}{|c|c|c|c|c|}
\hline Title & First Author & Year & Primary results & Conclusion \\
\hline $\begin{array}{l}\text { SIRT1/PGC1a-Dependent Increase in } \\
\text { Oxidative Phosphorylation Supports } \\
\text { Chemotherapy Resistance of Colon } \\
\text { Cancer }\end{array}$ & Thomas T.Vellinga & 2015 & $\begin{array}{l}\text { 1) Chemotherapy induces SIRT1 to } \\
\text { promote oxidative energy metabolism. } \\
\text { This gene controls mitochondrial } \\
\text { biogenesis by deacetylation and } \\
\text { activation of PGCla. 2) SIRT1 and } \\
\text { PGC1a protect colon cancer cells } \\
\text { against chemotherapy. }\end{array}$ & $\begin{array}{l}\text { Colorectal tumors shift their energy } \\
\text { metabolism when challenged with } \\
\text { chemotherapy. Chemotherapy } \\
\text { induces oxidative phosphorylation in } \\
\text { colon cancer cells via the SIRT1/ } \\
\text { PGC1a axis to help them survive } \\
\text { treatment. }\end{array}$ \\
\hline
\end{tabular}

AMPK Promotes Aberrant PGC1 $\beta$ Expression To Support Human Colon Tumor Cell Survival
Kurt W. Fisher

2015 PGC1a is not detected in HCT116 cell line. 1) PGC1 $\beta$ and ERRa are key downstream effectors of K-Ras, KSR1, and AMPK1. 2) Both AMPK 1 and K-Ras depletion decreased the protein levels of PGC1 $\beta$. 3) PGC1 $\beta$ and ERRa are required for colon cancer survival both in vivo and in vitro.

Peroxisome proliferator-activated receptor-ycoactivator 1-a (PGC1a) is a metabolic regulator of intestinal epithelial cell fate

Ilenia D’Errico

2011 1) Expression level of PGC1a in the intestine is higher in differentiated enterocytes than in the proliferative compartment at the bottom of the crypts, where it has only a scattered expression. 2) PGCla Induces Mitochondrial Proliferation and Activation in Human Intestinal Cancer Cells. 3) PGC1a induces tissue-specific accumulation of reactive oxygen species and apoptosis. 4) PGC1a Stimulates Intestinal Mitochondrial Biogenesis and Respiration in vivo, and suppresses Colorectal Carcinogenesis

Peroxisome Proliferator-Activated Receptor Coactivator-1alpha Enhances Antiproliferative Activity of 5'-Deoxy-5Fluorouridinein Cancer Cells through Induction of Uridine Phosphorylase
Xingxing Kong

2009 1) PGC-1 Induces the Expression of UPase in Breast and Colon Cancer Cells. 2) PGCla-Dependent Induction of UPase Gene in Cancer Cells Is Mediated by ERRa. 3) Overexpression of PGC-1 Sensitizes Cancer Cells to 5 -DFUR. overexpressed in colon cancer and are

The aberrant expression of $P G C 1 \beta$ and ERRa that persists in additional tumors with oncogenic Ras alleles will reveal the importance of these transcriptional regulators in creating tumor cells and promoting their survival. This may represent a new therapeutic target.

1) PGC1 a expression levels could influence intestinal epithelial cell fate by inducing mitochondrial-related metabolic modifications that induce apoptosis. 2) PGCla overexpression stimulates mitochondrial biogenesis, metabolic activities and accumulation of reactive oxygen species. 3) In tissues with high aerobic energy demand, PGCla preserves reactive oxygen species'homeostasis; In normal intestine, PGCla cannot induce reactive oxygen species scavenging systems.

1) PGC-1aseems to be a regulator of UPase gene transcription, whose effect is mediated by ERRa. 2) PGC1a has an effect on the absence of ERRa, suggesting the involvement of other regulatory factors. 3) In tumor cells, UPase catalyzes the transformation of 5 -DFUR to 5-FU, which inhibits their proliferation. In this way, PGCla enhances the cell's sensitivity to the treatment.

1) PPARB/ $\delta$ may repress PPARa and PPARY target gene expression. 2) relative to normat mucosa. 2) PGC-1 expression in the tumors was significantly decreased relative to normal mucosa.
RPARY target gene expression. 2)
Reduced coactivator levels ofPGC-1 are compatible with reduced transcriptional activity of PPARY and hence reduced tumor suppressor activity. 3) Transcriptional activity of PPARY may not only be decreased by mutation and increased levels of the transcriptional repressor PPAR $/ \delta$ but also by downregulation of coactivator PGC-1 of PPARY mitochondrial biogenesis pathway (via positive feedback), or chemotherapy.

In contrast, cancer development can also be promoted by silencing or downregulation of PGC1 $\alpha$, also via two possible mechanisms. First, low levels of PGC1 $\alpha$ hinder the correct function of the mitochondrial biogenesis pathway, which promotes CRC growth. Second, low levels of PGC1 $\alpha$ reduce PPAR- $\Upsilon$, which promotes CRC growth via an unknown process.
Figure 4 highlights some mechanisms that reduce CRC risk or development. In this case, reduction of PGC1 $\alpha$ levels is due to inhibition of KSR1 and AMPKҮ1 or XCT790 chemotherapy, via three possible mechanisms. First, lack of PGC1 $\alpha$ increases reactive oxygen species levels, which activates apoptosis. Second, reduced mitochondrial membrane potential $(\Delta \Psi \mathrm{m})$ reduces resistance to chemotherapy. Third, low expression of PGC1 $\alpha$ seems to reduce ERR $\alpha$ expression, reducing 
Table 2 Cell lines and other materials, and characteristics of articles considered in this review

\begin{tabular}{lll}
\hline Title & Main Material & Characteristics \\
\hline $\begin{array}{l}\text { Overexpression of PGC-1a enhances cell proliferation } \\
\text { and tumorigenesis of HEK293 cells through the }\end{array}$ & HT-29 (Cell line) & i) Human. \\
upregulation of Sp1 and Acyl-CoA binding protein & SNU-C4 (Cell line) & ii) Epithelial \\
& CT-26 (Cell line) & i) Human \\
& & i) Mouse. \\
PGC-1 $\beta$ promotes enterocyte lifespan and & iPGC1b mouse model with human PGC1b & ii) Epithelial
\end{tabular}

tumorigenesis in the intestine

Mitochondria and Tumor Progression in Ulcerative Colitis

PGC1a promotes tumor growth by inducing gene expression programs supporting lipogenesis.

Bax is necessary for PGCla pro-apoptotic effect in colorectal cancer cells

PGC -1 a/ $\beta$ upregulation is associated with improved oxidative phosphorylation in cells harboring nonsense mtDNA mutations

Validation of the Use of DNA Pools and Primer Extension in Association Studies of Sporadic Colorectal Cancer for Selection of Candidate SNPS

SIRT1/PGC1a-Dependent Increase in Oxidative Phosphorylation Supports Chemotherapy Resistance of Colon Cancer

AMPK Promotes Aberrant PGC1b Expression To Support Human Colon Tumor Cell Survival

Peroxisome proliferator-activated receptor- $\gamma$ coactivator 1-a (PGC1a) is a metabolic regulator of intestinal epithelial cell fate

Peroxisome Proliferator-Activated Receptor gamma Coactivator-1 Enhances Antiproliferative Activity of 5 -Deoxy-5-Fluorouridine in Cancer Cells through Induction of Uridine Phosphorylase
PGC1b mouse model with human PGC1b

iPGC1b knockout mice

iPGC1b Apc Min/+ Mice

Ulcerative Colitis progressor (Human)

i) Human

Ulcerative Colitis non progressor (Human)

PGCla knockout mice

i) Transgenic mouse

PGC1a +/+ mice

SCID mice (HT29)

i) Inoculated mouse

HT29 (Cell line)

Colo205 (Cell line)

i) Human.

ii) Epithelial

i) Human.

ii) Epithelial

HCT116 (Cell line)

Nude mice (subcutaneously injected both cells)

i) Human

ii) Epithelial

i) Inoculated mouse

VACO425 (Cell line)

i) Human

VACO429 (Cell line)

i) Human

Two pools of genomic DNA (patients with sporadic CRC + controls)

i) Human

Colonosphere cultures shSIRT1 (Human colorectal tumor specimens)

i) Human colonosphere cultures

Colonosphere cultures shPGC1a (Human colorectal tumor specimens)

HCT116 (Cell line)

i) Human.

ii) Epithelial

Inmunodeficient mice (HCT116 cells grafted into mice)

HT29 (Cell line)

i) Inoculated mouse

i) Human.

ii) Epithelial

HCT116 (Cell line)

i) Human.

ii) Epithelial

HT29p0 (Cell line: Completely lacks mitochondrial DNA)

Xenograft mice using HT29 cells

i) Human.

ii) Epithelial

i) Inoculated mouse

i) Transgenic mouse

PGC1a +/+ mice and PGC1-/- mice

Colo320 (Cell line)

i) Human.

ii) Undifferentiated

i) Human.

ii) Epithelial 
Table 2 Cell lines and other materials, and characteristics of articles considered in this review (Continued)

\begin{tabular}{lll}
\hline Title & Main Material & Characteristics \\
\hline Peroxisome proliferator-activated receptors & Colorectal cancers from patients (Human) & i) Human \\
(PPARs) and associated transcription factors in & & \\
colon cancer: reduced expression of PPARg- & & \\
coactivator 1 (PGC-1) & & \\
\hline
\end{tabular}

CRC. In contrast, other mechanisms support the idea that PGC1 $\alpha$ overexpression increases ERR $\alpha$ expression, which may increase chemotherapy sensitivity.

These contrasting results could be due to the different starting material (e.g. cell lines, mice, etc.) in these studies. In Table 2 we summarize the material used in each article with the objective of trying to understand the cause of these differences. Note that we only indicate the main starting material, from which the authors obtained the principal results.

\section{Checking the synthesis with authors of primary studies}

We were unable to compare our results to those of other systematic reviews because no other reviews have dealt with this topic, as far as we are aware.

\section{Discussion}

The central question of this review is clear: summarize current evidence on the role of PGC1 $\alpha$ in the initiation and progression of CRC. We tested the effect of different PGC1 $\alpha$ expression levels during the development of colorectal cancer. There is little prior evidence on this question, so only a small number of studies have been included in this review.

The studies included in this review aimed to evaluate whether an increase or decrease in PGC1 $\alpha$ expression levels affects the development of CRC. Most studies $(61.5 \%)$ were carried out using cell lines [10, 11, 20, 21, 23-25]: in four studies, cells were injected subcutaneously into the flanks of mice [20, 21, 23, 24], and two studies evaluated expression levels in mice in vivo $[14,21]$. Other studies were carried out in samples of patients with disease $[6,13,26,27]$, with similar results to the in vitro studies.

Each article proposes a possible molecular mechanism of action. First, we try to understand the normal mechanism of PGC1 $\alpha$ action proposed by Ilenia D'Errico et al. [20], which is derived from work on cell cultures and two groups of transgenic mice: iPGC1 $\alpha$ transgenic mice and iPGC1 $\alpha \mathrm{Apc}^{\mathrm{Min} /+}$ mice (more information in Table 2). The authors conclude that PGC1 $\alpha$ is a metabolic regulator of intestinal cell fate. In addition, PGC1 $\alpha$ seems to have different functions in tissues with high aerobic energy metabolism, such as at the bottom of the crypts, where PGC1 $\alpha$ expression increases production of

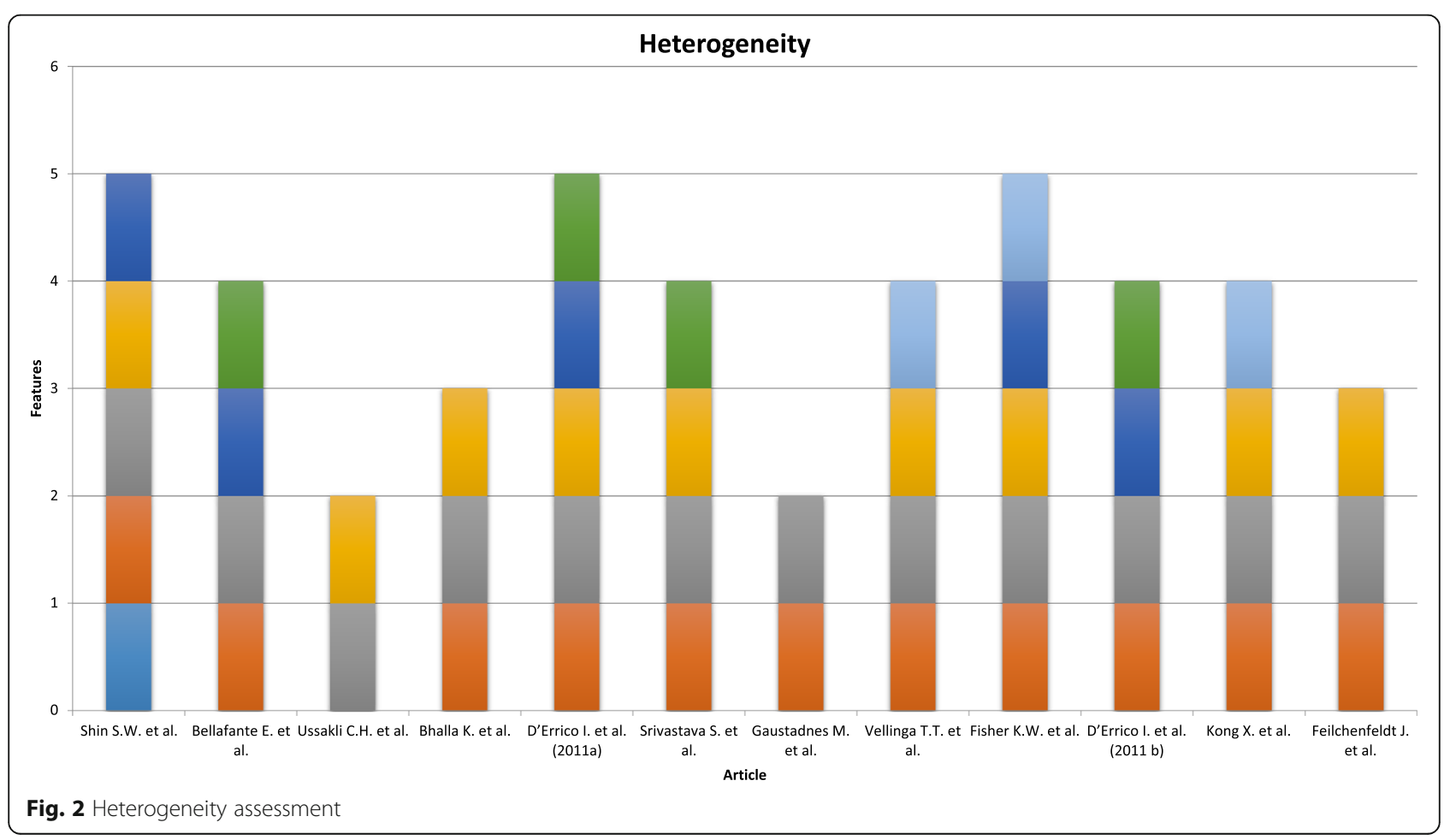


Normal conditions. Constitutive PGC1 $\alpha$ expression in normal epithelium [20]

a

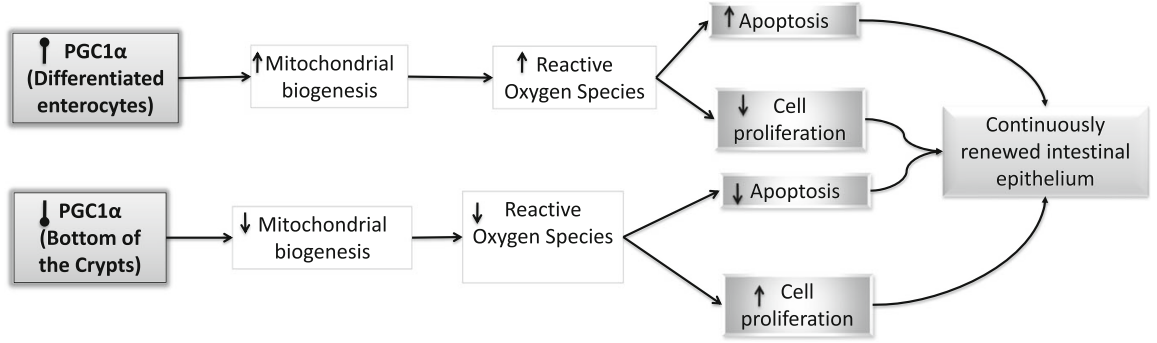

Overexpression PGC1 $\alpha$ in human colon cancer cells (HT29 cells) [20]

b

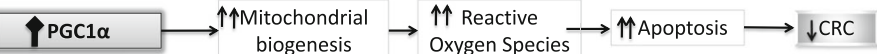

Fig. 3 Concept map based on D'Errico et al. Ref [20]

antioxidant enzymes that protect cells from reactive oxygen species. In contrast, at the top of the villi, where aerobic energy metabolism is low, PGC1 $\alpha$ expression increases mitochondrial biogenesis, resulting in greater accumulation of reactive oxygen species than at the bottom of the crypts, which induces apoptosis [20]. In this way, there is a balance between cell proliferation and apoptosis under normal conditions.

Other mechanisms have been proposed on the basis of various studies of $\mathrm{PGC1} \alpha$ overexpression under nonnormal conditions, mainly in cell culture. One mechanism supports the idea that overexpression of PGC1 $\alpha$ results in high levels of $\mathrm{Sp} 1$ (specificity protein 1 ), which is then thought to enhance expression of ACBP (Acyl-
CoA-binding protein) via ACBP's Sp1 binding site. ACBP upregulation increases cell proliferation and decreases sensitivity to $\mathrm{H} 2 \mathrm{O} 2$-induced apoptosis $[10,14]$. A second mechanism proposes that PGC1 $\alpha$ is a key metabolic regulator of several aspects of glucose metabolism. PGC1 $\alpha$ is thought to coordinate gene expression in metabolic pathways that convert glucose to fatty acids. In turn, fatty acid synthesis promotes tumor growth [21] and disrupts the balance between apoptosis and cell proliferation, promoting the development of colorectal cancer.

A third possible mechanism is based on overexpression of both PGC1 $\alpha$ and PGC1 $\beta$, which increases the electron transfer activity of the mitochondrial respiration

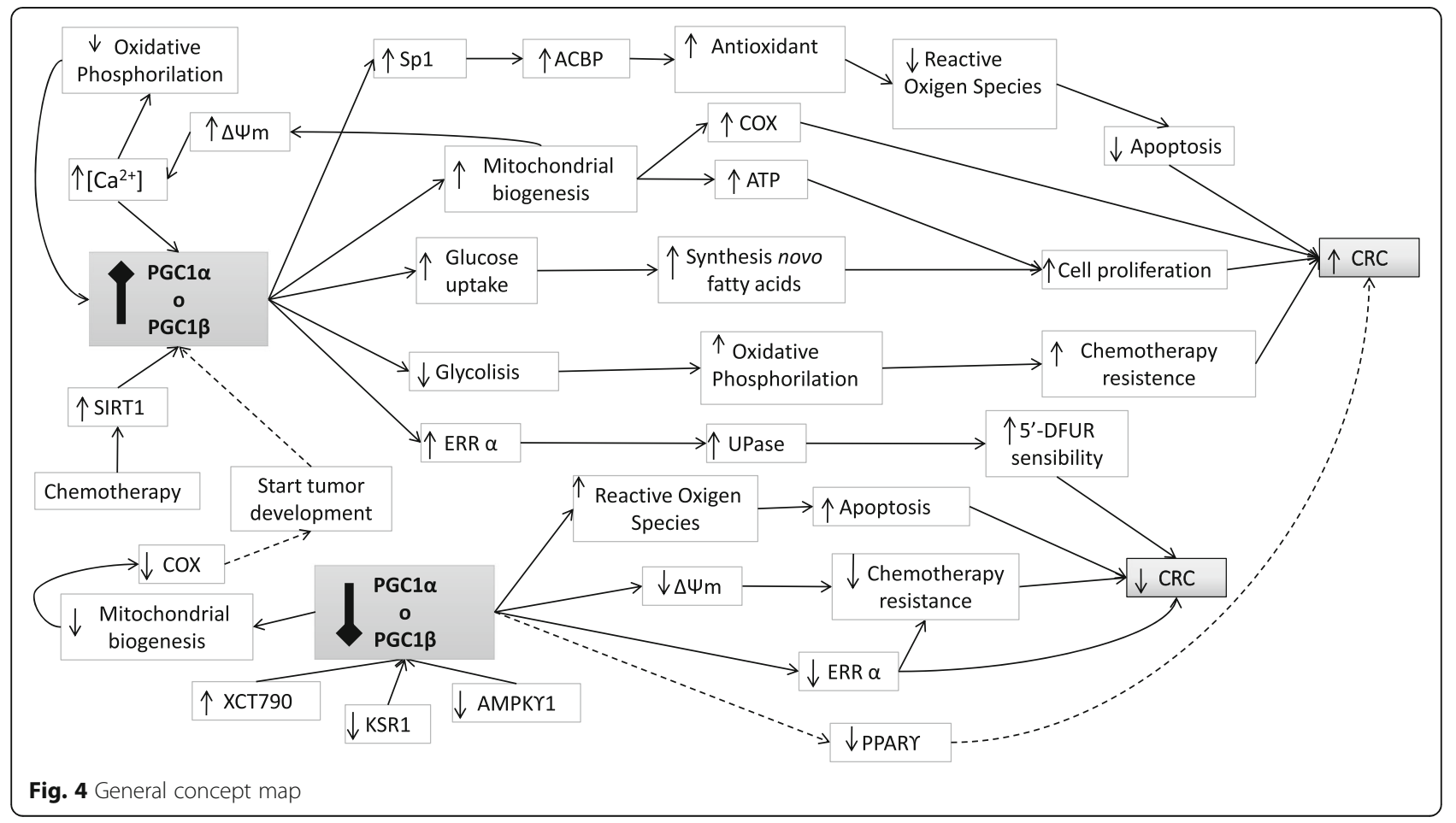


chain and augments mitochondrial biogenesis [11, 14]. Both processes partly disrupt $\Delta \Psi \mathrm{m}$ and cytosolic calcium [Ca2+] buffering ability, with two consequences: 1 ) Dysfunctional oxidative phosphorylation in mitochondria, and 2) stimulation of the $\mathrm{Ca} 2+$ signaling cascade, which in turn may activate genes involved in tumor invasion and metastasis. Both of these effects can upregulate PGC1 $\alpha$, generating a positive feedback loop [11]. In addition, the increase in mitochondrial biogenesis seems to give the cells the necessary energy for increased longevity and cellular division, promoting tumor growth [11, 14].

From these three molecular mechanism we can conclude that PGC1 $\alpha$ and PGC1 $\beta$ allow cells to balance i) mitochondrial activity and cytotoxic protection in the production of reactive oxygen species, and ii) apoptosis and cell proliferation $[10,11,14,21]$. In addition, we can observe that all studies in cell lines $[10,11,21]$ and mice [14] support the idea that high expression of PGC1 $\alpha$ carries increased CRC risk. Note that while none of these articles work with the same cell line, the cells used are similar since the majority of cell lines are epithelial (Table 2). Shin et al. [10] use human colon cancer cells (HT29 and SNU-C4) and mouse colon cancer cells (CT26). Srivastava et al. [11] use VACO 425 and VACO429, another type of human colon cancer cell. Bhalla et al. [21] use other human colon adenocarcinoma cells, Colo205, as well as HT29, like Shin et al. Despite this, all of these studies arrived at the same conclusions.

However, Ilenia D'Errico [24] show that Bax is necessary for the pro-apoptotic effect of PGC1 $\alpha$ in colorectal cancer cells, which could be another molecular mechanism of action, although this is not consistent with other studies. In this study [24], PGC1 $\alpha$ overexpression seems to induce Bax translocation to mitochondria, and Bax-protein mediates the pro-apoptotic effect of PGC1 $\alpha$ [24]. In this case, the cell line used is HCT116 (human colon carcinoma, also epithelial cells) and they also use mice who were subcutaneously injected with these cells. Here there is some controversy: in absence of Bax, PGC1 $\alpha$ overexpression was not able to oppose tumor growth.

In addition to this research on overexpression of PGC1a, other studies have focused on low expression, inhibition or silencing of PGC1 $\alpha$ and PGC1 $\beta$. Using the same culture cells as Ilenia D'Errico [24], as well as other cells and in inmunodeficient mice, Fisher et al. [23] showed that PGC1 $\beta$ is aberrantly expressed in human colon cell lines and tumors, and maintains $E R R \alpha$ levels, contributing to its tumorigenic properties. These authors also found that KSR1 (Kinase suppressor of Ras 1) and AMPK (AMP-activated protein kinase), which act upstream of the PGC1 $\beta$ promotor, were linked to the action of transcriptional regulators PGC1 $\beta /$ ERR $\alpha$ (estrogen-related receptor $\alpha$ ). Reduced
KSR1 and AMPK expression also downregulated expression of PGC1 $\beta$ and ERR $\alpha$, which inhibits the survival of colorectal cancer cells [23].

Based on all of this evidence, it seems that PGC1 $\alpha /$ PGC1 $\beta$ is overexpressed in human colon cell lines during tumor development, and this overexpression seems to be required for both cell proliferation and survival, and to reduce apoptosis [10,11, 14, 21, 23]. Only one study obtained markedly different results despite using the same cell line as one of the above (HCT116), although this was based on Bax instead of PGC1 $\alpha$ [24]. Note that while all of these studies were performed using different cell lines with genetic changes, most of these are human epithelial cells (Table 2). For this reason, different results could not be attributed to the use of different cell lines. We consider that we cannot explain the origin of this differences.

Our systematic search returned two studies that analyzed the effects of chemotherapy on PGC1 $\alpha$ expression in cultured cells and colonosphere cultures (Table 2) $[25,26]$. These studies suggest three other molecular mechanism of action of PGC1 $\alpha .1)$ Chemotherapy of colorectal tumor cells induces a SIRT1/PGC1adependent increase in oxidative phosphorylation that promotes tumor survival during treatment because chemotherapy induces a shift in tumor energy metabolism that protects tumor cells from cytotoxic damage [26]. Mechanistically, chemotherapy-induced DNA damage results in increased expression of SIRT1, which deacetylates and thereby activates PGC1 $\alpha$ as a transcriptional coactivator. PGCla acts in concert with several transcription factors to stimulate the expression of genes involved in mitochondrial biogenesis and respiration, resulting in increased oxidative phosphorylation and helping them survive treatment. In this case, the authors worked with human colonosphere cultures [26]. 2) PGC1 $\alpha$-induced activation of Uridine phosphorylase (UPase) expression, which is mediated by an estrogen related receptor (ERR) binding site. Overexpression of PGC1 $\alpha$ via this mechanism sensitizes colon cancer cells to growth inhibition by 5-deoxy-5-fluorouridine, presumably by inducing apoptosis in tumor cells [25]. In this way, 3) ERR $\alpha$ is primarily thought to regulate energy homeostasis by interacting with PGC1 $\alpha$ or PGC1 $\beta$. $E R R \alpha$ overexpression reduces sensitivity to chemotherapy, while inhibition of these genes reduces reactive oxygen species and $\Delta \Psi \mathrm{m}$, which increases sensitivity to chemotherapy [22, 23]. Here, Fisher et al. worked with HCT116 and other cell lines [23], but studied the effect of the chemotherapy on HepG2 [22].

These mechanisms support the conclusion mentioned above, that PGC1 $\alpha$ and PGC1 $\beta$ allow cells to balance i) mitochondrial activity and cytotoxic protection in the production of reactive oxygen species, and ii) apoptosis 
and cell proliferation. In addition, they suggest that any therapy that reduces PGC1 $\alpha$ expression will increase cancer cells' sensitivity to chemotherapy. Thus, more and more authors support the idea of using PGC1 $\alpha$ as a potential target in cancer therapy [21-23, 25, 26]. In fact, Do et al. [28] reported a strategy to reduce PGC1 $\alpha$ levels to improve cancer therapy, though not in colon cancer cells. They showed for the first time that metformin, an insulin-lowering agent [29], induces miR-34a which reduces Sirt1 in wild-type p53 cancer cells, but does not occur in altered p53 cell lines. This fact was shown in HCT116 and MCF-7 cell lines among others. However, they only could show in an MCF-7 cell line (Breast cancer cells) that the reduction of Sirt1 involved reduced PGC1 $\alpha$ and, subsequently, reduced NRF2 and enhanced susceptibility to oxidative stress [28]. DeCensi et al. 2010 [29] showed using a meta-analysis that metformin is associated with decreased risk of cancer, including colon cancer, in diabetic patients compared with other treatments [29].

Our systematic search returned three articles based on human samples. First, Ussakli et al. [6] studied 9 non-dysplastic colon biopsies from Ulcerative Colitis (UC) patients with high-grade dysplasia or cancer and 9 dysplasia-free UC patients, and concluded that while the development of dysplasia is preceded by mitochondrial loss, mitochondria are restored in cancer cells, which suggests that they are needed for further proliferation. This bimodal pattern may be driven by transcriptional regulation of mitochondrial biogenesis by PGC1 $\alpha$ [6], which is consistent with the results of the cell culture studies described above [11, 14].

Two other papers propose the same idea, that overexpression of PGC1 $\alpha$ results in higher CRC risk, although these studies are not comparable with the one described above [6]. First, Gaustadnes et al. [27] screened a selection of SNPs in pooled DNA, and found that the rs96516 SNP in the PPARGC-1A ("604517) 3’UTR was significantly associated with sporadic CRC risk, although this result was not reproducible in the same study [27]. Second, Feilchenfeldt et al. [13] studied expression levels of all isoforms of PPAR- $\Upsilon$ and transcriptional partners such as PGC1 $\alpha$ in patients with different stages of colon cancer, and found that expression levels of PPAR- $\Upsilon$ vary between isoforms and cancer stages, while those of PGC1 were reduced in all cancer samples, with respect to normal samples [13].

Summarizing, while D'Errico et al., 2011 [20] showed that PGC1 $\alpha^{-/-}$mice are susceptible to intestinal tumorigenesis, several papers addressing the role of PGC-1 in tumor cell lines showed a role of PGC-1 in tumor progression. Thus, the function of PGC1 $\alpha$ in colorectal cancer risk is not entirely clear, although it seems likely that it has a role in this disease.

\section{Conclusions}

Colorectal cancer is a major global public health problem and the second leading cause of cancer-related death. Our systematic review indicates that altered expression of PGC1 $\alpha$ modifies CRC risk. Most studies showed that overexpression of this gene increases CRC risk $[10,11,14,21,23,25,26]$, while some studies indicated that lower than normal expression levels could increase CRC risk $[6,13]$. Thus, various authors suggest that PGC1 $\alpha$ is a good candidate as a molecular target for cancer therapy. Reducing expression of this gene could help to reduce risk or progression of CRC $[22,25,26]$, at least in different cell lines and transgenic or nude mice. However, in our opinion, there are not enough data on the role of PGC1 $\alpha$ using human tumor samples to conclude a role of this gene in CRC. This question should be investigated further.

\section{Additional file}

Additional file 1: Table S1. Reasons for exclusion of 22 articles according to abstract and full-text. Table that describes the reasons for exclusion of the selected articles in the first review. Table S2. Full details of the key characteristics of the selected studies. Table that describes the details of the selected articles. (PDF $245 \mathrm{~kb}$ )

\section{Abbreviations}

ACBP: Acyl-CoA-binding protein; AMPK: AMP-activated protein kinase; CRC: Colorectal cancer; ERRa: Estrogen-related receptor a; Fig.: Figure; KSR1: Kinase suppressor of Ras 1; PGC-1: Peroxisome proliferator-activated receptor gamma coactivator 1; PPARGC1A or PGC1a: Peroxisome proliferatoractivated receptor gamma coactivator 1-a; Sp1: Specificity protein 1; UC: Ulcerative Colitis; UPase: Uridine phosphorylase; $\Delta \psi \mathrm{m}$ : Mitochondrial membrane potential

\section{Acknowledgements \\ We thank Silivia Gutierrez and Verónica Dávila their help with methodological and intellectual contributions to the review.}

\section{Funding}

No funding was received for this research.

\section{Availability of data and materials}

All data generated or analyzed during this study are included in this published article and its supplementary information files.

\section{Authors' contributions}

JAM: First author and corresponding author. Her functions: To search for a protocol to make the review, make the online review, check titles, check abstracts, check full text and write the review. She made substantial contributions to conception and design, acquisition of data, analysis and interpretation of data. In addition, she has been involved in drafting the manuscript and revising it critically for important intellectual content and she agreed to be accountable for all aspects of the work by ensuring that questions related to the accuracy or integrity of any part of the work are appropriately investigated and resolved. CGD: Second author. Expert in the subject of the review. Her functions: To make the online review, check titles, check abstracts and check full text. She made substantial contributions to acquisition of data, analysis and interpretation of data. TFV: Third Author. Not 
an expert in the subject of the review. Her functions: To make the online review and check titles. She made substantial contributions to acquisition and analysis of data. FST: Fourth author. Expert in the subject of the review. Her functions: To check the review. She has been involved in revising the manuscript critically for important intellectual content. LVM: Fifth author. Expert in the subject of the review. Her functions: To check the review. She has been involved in revising the manuscript critically for important intellectual content. AJM: Sixth author. Not an expert in the subject of the review. His functions: To check the review. He has been involved in revising the manuscript critically for important intellectual content. VMS: Senior author. His functions: To approve the protocols to be followed in the study, perform manuscript correction and proof reading. He agreed to be accountable for all aspects of the work by ensuring that questions related to the accuracy or integrity of any part of the work are appropriately investigated and resolved. All of the authors have participated sufficiently in the work to take public responsibility for appropriate portions of the content. All of them have read and approved the final manuscript.

\section{Ethics approval and consent to participate}

Not applicable.

\section{Consent for publication}

Not applicable.

\section{Competing interests}

The authors declare that they have no competing interests.

\section{Publisher's Note}

Springer Nature remains neutral with regard to jurisdictional claims in published maps and institutional affiliations.

\section{Author details}

${ }^{1}$ Grupo de Investigación en Interacciones Gen-Ambiente y Salud, Universidad de León, León, Spain. ${ }^{2}$ Universidad de Cantabria, Santander, Spain. ${ }^{3}$ Superior Physical Education School, Federal University of Pelotas, Pelotas, Brazil. ${ }^{4} \mathrm{CIBER}$ Epidemiología y Salud Pública (CIBERESP), Madrid, Spain. ${ }^{5}$ Departamento Medicina Preventiva y Salud Pública, Facultad Ciencias de la Salud, Campus Vegazana, s/n. León, C.P.: 24071 Castilla y León, Spain.

Received: 29 November 2016 Accepted: 30 October 2017

Published online: 09 November 2017

\section{References}

1. Ferlay J, Shin HR, Bray F, Forman D, Mathers C, Parkin DM. Estimates of worldwide burden of cancer in 2008: GLOBOCAN 2008. Int J Cancer. 2010; 127:12.

2. Vega P, Valentín F. Cubiella. J Colorectal cancer diagnosis: Pitfalls and opportunities World J Gastrointest Oncol. 2015;7:12.

3. Abraha I, Giovannini G, Serraino D, Fusco M, Montedori A. Validity of breast, lung and colorectal cancer diagnoses in administrative databases: a systematic review protocol. BMJ Open. 2016;6:3.

4. Smolková K, Plecitá-Hlavatá L, Bellance N, Benard G, Rossignol R, Ježek P. Waves of gene regulation suppress and then restore oxidative phosphorylation in cancer cells. Int J Biochem Cell Biol. 2011;43:7.

5. Bianchi G, Martella R, Ravera S, Marini C, Capitanio S, Orengo A, et al. Fasting induces anti-Warburg effect that increases respiration but reduces ATP-synthesis to promote apoptosis in colon cancer models. Oncotarget. 2015;6:14

6. Ussakli CH, Ebaee A, Binkley J, Brentnall TA, Emond MJ, Rabinovitch PS, et al. Mitochondria and tumor progression in ulcerative colitis. J Natl Cancer Inst. 2013;105:16.

7. Jose C, Bellance N, Rossignol R. Choosing between glycolysis and oxidative phosphorylation: a tumor's dilemma? Biochim Biophys Acta Bioenerg. 2011; 1807:6.

8. Jones AWE, Yao Z, Vicencio JM, Karkucinska-Wieckowska A, Szabadkai G. PGC-1 family coactivators and cell fate: roles in cancer, neurodegeneration, cardiovascular disease and retrograde mitochondria-nucleus signalling. Mitochondrion. 2012;12:1.

9. Mazzanti R, Giulivi C. Coordination of nuclear- and mitochondrial-DNA encoded proteins in cancer and normal colon tissues. Biochim Biophys Acta Bioenerg. 2006;1757:5-6.
10. Shin SW, Yun SH, Park ES, Jeong JS, Kwak JY, Park JI. Overexpression of PGC-1a enhances cell proliferation and tumorigenesis of HEK293 cells through the upregulation of Sp1 and acyl-CoA binding protein. Int J Oncol. 2015:46:3.

11. Srivastava S, Barrett JN, Moraes CT. PGC-1 1 / $\beta$ upregulation is associated with improved oxidative phosphorylation in cells harboring nonsense mtDNA mutations. Hum Mol Genet. 2007;16:8

12. Villena JA. New insights into PGC-1 coactivators: redefining their role in the regulation of mitochondrial function and beyond. FEBS J. 2015;282:4.

13. Feilchenfeldt J, Bründler MA, Soravia C, Tötsch M, Meier CA. Peroxisome proliferator-activated receptors (PPARs) and associated transcription factors in colon cancer: reduced expression of PPARy-coactivator 1 (PGC-1). Cancer Lett. 2004;203:1.

14. Bellafante $\mathrm{E}$, et al. PGC-1 $\beta$ promotes enterocyte lifespan and tumorigenesis in the intestine. Proc Natl Acad Sci U S A. 2014;111:42.

15. Seale P. Transcriptional regulatory circuits controlling brown fat development and activation. Diabetes. 2015;64:7.

16. López-Lluch G, Irusta PM, Navas P, De Cabo R. Mitochondrial biogenesis and healthy aging. Exp Gerontol. 2008;43:9.

17. Van Gisbergen MW, Voets AM, Starmans MHW, De Cood IFM, Yadakd R, Hoffmanne RF, et al. How do changes in the mtDNA and mitochondrial dysfunction influence cancer and cancer therapy? Challenges, opportunities and models. Mutat res. Rev Mutat Res. 2015;764:16-30.

18. Popay J, Roberts H, Sowden A, Petticrew M, Arai L, Rodgers M, et al. Guidance on the conduct of narrative synthesis in systematic reviews. Biostats. 1995:536(22):3.

19. Moher D, Liberati A, Tetzlaff J, Altman DG, PRISMA GROUP. Preferred Reporting. Items for systematic reviews and meta-analyses: the PRISMA statement. Ann Intern Med. 2009;6(7):264-9.

20. D'Errico I, Salvatore L, Murzilli S, Lo Sasso G, Latorrec D, Martelli N, et al. Peroxisome proliferator-activated receptor-gamma coactivator 1-alpha (PGC1alpha) is a metabolic regulator of intestinal epithelial cell fate. Proc Natl Acad Sci U S A. 2011:108:16

21. Bhalla K, Hwang BJ, Dewi RE, Ou L, Twaddel W, Fang H, et al. PGC1a promotes tumor growth by inducing gene expression programs supporting lipogenesis. Cancer Res. 2011;71:21.

22. Wu F, Wang J, Wang Y, Kwok TT, Kong SK, Wong C. Estrogen-related receptor a (ERRa) inverse agonist XCT-790 induces cell death in chemotherapeutic resistant cancer cells. Chem Biol Interact. 2009;181:2.

23. Fisher KW, Das B, Seok Kim H, Clymer BK, Gehring D, Smith DR, et al. AMPK Promotes Aberrant PGC1 $\beta$ Expression to Support Human Colon Tumor Cell Survival. 2015:35:22

24. D’Errico I, Lo Sasso G, Salvatore L, Murzilli S, Martelli N, Cristofaro M, et al. Bax is necessary for PGC1a pro-apoptotic effect in colorectal cancer cells. Cell Cycle. 2011;10:17.

25. Kong X, Fan H, Liu X, Wang R, Liang J, Gupta N, et al. Peroxisome proliferator-activated receptor gamma Coactivator-1 alpha enhances Antiproliferative activity of 5J-Deoxy-5-Fluorouridine in cancer cells through induction of uridine phosphorylase. Mol Pharmacol. 2009;76:4.

26. Vellinga TT, Borovski T, de Boer VCJ, Fatrai S, Van Schelven S, Trumpi $K$, et al. SIRT1/PGC1a-dependent increase in oxidative phosphorylation supports chemotherapy resistance of colon cancer. Clin Cancer Res. 2015;21:12.

27. Gaustadnes M, Ørntoft TF, Jensen JL, Torring N. Validation of the use of DNA pools and primer extension in association studies of sporadic colorectal cancer for selection of candidate SNPs. Hum Mutat. 2006;27:2.

28. Do MT, Kim HG, Choi JH, Jeong HG. Metformin induces microRNA-34a to downregulate the Sirt1/Pgc-1a/Nrf2 pathway, leading to increased susceptibility of wild-type p53 cancer cells to oxidative stress and therapeutic agents. Free Radic Biol Med. 2014;74:21-34.

29. DeCensi A, Puntoni M, Goodwin P, Cazzaniga M, Gennari A, Bonanni B, Gandini S. Metformin and cancer risk in diabetic patients: a systematic review and meta-analysis. Cancer Prev Res. 2010;3:1451-61. 